\title{
DISTANT RADIO GALAXIES: THE STRONG LINK BETWEEN THE RADIO AND OPTICAL EMISSION.
}

\author{
HUUB RÖTTGERING \\ Leiden Observatory, The Netherlands
}

\begin{abstract}
Recent observations of distant radio galaxies show that there is a strong link between the radio source and the optical continuum and Ly $\alpha$ line emission from the galaxy. This link is discussed in terms of differences in age, orientation and environment between the radio sources.
\end{abstract}

The double peaked velocity structure of the $\operatorname{Ly} \alpha$ emission of the radio source $0943-242(z=2.9)$ is likely to be caused by extended regions (e.g. $>$ $13 \mathrm{kpc}$ ) of neutral hydrogen with column densities of $10^{19} \mathrm{~cm}^{-2}$ (Röttgering et al. 1995).

The Ly $\alpha$ gas associated with the radio galaxy $1243+036(z=3.6)$ has three distinct components (van Ojik et al. 1995a): (i) gas with a high velocity dispersion ( $1550 \mathrm{~km} \mathrm{~s}^{-1} \mathrm{FWHM}$ ) located inside the radio structure, (ii) enhanced Ly $\alpha$ emission blue-shifted by $1100 \mathrm{~km} \mathrm{~s}^{-1}$ at the location of the strong bend in the radio jet and (iii) Ly $\alpha$ emission extending out well beyond the radio lobes. This emission has a low velocity dispersion (250 $\mathrm{km} \mathrm{s}^{-1}$ FWHM) and a velocity gradient of $450 \mathrm{~km} \mathrm{~s}^{-1}$ over the extent of the emission, indicative of large scale rotation. We advocate a scenario for the formation of this object in which the outer halo is associated with the accretion of gas during the formation of the galaxy.

The results on these 2 radio galaxies prompted us to study the $\mathrm{HI}$ absorption and dynamics of the Ly $\alpha$ gas for a sample of distant radio galaxies (van Ojik, 1995; van Ojik et al. 1995b). Here we briefly summarize the results. (1) In 11 radio galaxies from a sample of 18 we find strong ( $>10^{18}$ $\mathrm{cm}^{-2}$ ) H I absorption. (2) The radio sources larger than about $50 \mathrm{kpc}$ do not show strong absorption; almost all the smaller ones do. Other clear indications that the radio source size and the Ly $\alpha$ emission are closely linked are that (3) higher velocity dispersions in the Ly $\alpha$ gas are found in the smaller 
radio sources and (4) larger radio sources tend to have larger regions of Ly $\alpha$ emission. (5) The amount of the distortion in the Ly $\alpha$ gas strongly correlates with the amount of distortion in the radio sources. (6) It seems that - at least in some sources - there are 2 components in the Ly $\alpha$ halo; an inner halo located within the boundaries of the radio source, that has a high velocity dispersion $\left(700-1600 \mathrm{~km} \mathrm{~s}^{-1}\right)$ and an outer halo located outside the radio source that has a low velocity dispersion $\left(\sim 300 \mathrm{~km} \mathrm{~s}^{-1}\right)$.

Finally, (7) the optical morphologies as observed by HST of a complete sample of $3 \mathrm{CR}$ radio galaxies in the redshift interval $1 \lesssim z \lesssim 1.3$ are highly dependent upon their radio properties (Best et al. 1995). There is a clear evolution of the optical structures as the size of the radio source increases: small radio sources consist of many bright knots, tightly aligned along the radio axis, whilst more extended sources contain fewer (generally no more than two) bright components and display more diffuse emission.

It seems difficult to understand these trends as reflecting differences in orientation. For example, in such a scenario it is not clear why smaller radio sources show such a pronounced absorption. It is possible to explain most of the trends through a scenario in which the smallest radio sources are in the densest environments. In such a dense environment there is a lot of neutral gas around the small radio sources to absorb the Ly $\alpha$ emission. The radio source heavily interacts with the dense gas leading to disturbed radio morphologies and relatively small radio source sizes. The large differences in radio source size within the $3 \mathrm{CR}$ sample has to be at least partly due to ageing of the radio source. We therefore conclude that it is likely that both the environment as well as the age of the radio source determine observed differences in these samples of distant radio sources.

Acknowledgements. I would like to thank my collaborators, Philip Best, Malcolm Bremer, Chris Carilli, Dick Hunstead, Malcolm Longair, George Miley and Rob van Ojik for the numerous discussions.

\section{References}

Best P., Longair M. S., Röttgering H. J. A., 1995, Evolution of the aligned structures in $z \sim 1$ radio galaxies, MN: submitted

Röttgering H., Hunstead R., Miley G. K., van Ojik R., Wieringa M. H., 1995, MNRAS, 277, 389

van Ojik R., 1995, Ph.D. thesis, University of Leiden

van Ojik R., Röttgering H., Carilli C., Miley G., Bremer M., 1995a, A radio galaxy at $z=3.6$ in a giant rotating Lyman $\alpha$ halo, $A \& A$ : in press

van Ojik R., Röttgering H. J. A., Miley G. K., Hunstead R., 1995b, The Gaseous Environment of Radio Galaxies in the Early Universe: Kinematics of the Lyman $\alpha$ Emission and Spatially Resolved HI Absorption, $A \mathcal{E} A$ : submitted 